Relations industrielles

Industrial Relations

\title{
Au-delà des frontières, par le Conseil Économique du Canada, Ottawa, Information Canada, 1975, XV + 226 p.
}

\section{Carmine Nappi}

Volume 30, numéro 4, 1975

URI : https://id.erudit.org/iderudit/028667ar

DOI : https://doi.org/10.7202/028667ar

Aller au sommaire du numéro

Éditeur(s)

Département des relations industrielles de l'Université Laval

ISSN

0034-379X (imprimé)

1703-8138 (numérique)

Découvrir la revue

Citer ce compte rendu

Nappi, C. (1975). Compte rendu de [Au-delà des frontières, par le Conseil Économique du Canada, Ottawa, Information Canada, 1975, XV + 226 p.] Relations industrielles / Industrial Relations, 30(4), 797-799.

https://doi.org/10.7202/028667ar

Tous droits réservés @ C Département des relations industrielles de l'Université Laval, 1975
Ce document est protégé par la loi sur le droit d'auteur. L'utilisation des services d'Érudit (y compris la reproduction) est assujettie à sa politique d'utilisation que vous pouvez consulter en ligne.

https://apropos.erudit.org/fr/usagers/politique-dutilisation/ 


\section{RECENSIONS BOOK REVIEWS}

Au-delà des frontières, par le Conseil Economique du Canada, Ottawa, Information Canada, 1975, XV +226 p.

Ce rapport du Conseil Economique désire démontrer que le problème majeur du secteur manufacturier canadien réside dans l'absence d'échelle et de spécialisation. Bien plus, la faiblesse de sa productivité est causée par les politiques commerciales adoptées par le Canada et les autres pays, en particulier par les mesures protectionnistes utilisées par le Canada depuis la Politique $\mathrm{Na}$ tionale de 1879. Ceci dit, le Conseil se donne comme tâche de démontrer que les objectifs économiques du Canada (augmenter le niveau de vie réel, réaliser le plein emploi, assurer une stabilité raisonnable des prix, promouvoir une distribution plus juste des revenus, soutenir la croissance de l'économie mondiale et accélérer les progrès des pays en voie de développement) peuvent plus facilement être atteints par une politique de libre-échange (chapitres 2 à 6). Les différentes formes de cette nouvelle politique commerciale, ainsi que leurs effets respectifs, sont discutés dans les chapitres 7 (la libération multilatérale), 8 (le libre-échange unilatéral et régional: Canada - U.S.A. - C.E.E. Japon, Canada, U.S.A. - C.E.E., Canada - U.S.A. - Japon), 9 (le libre-échange avec les Etats-Unis) et 10 (une approche sectorielle à la libération des échanges). Après avoir abordé les problèmes spécifiques des produits agricoles (chapitre 11) et énergétiques (chapitre 12) dans un contexte plus libreéchangiste, le rapport présente dans son chapitre 13 des mesures spécifiques d'aide gouvernementale et des politiques d'encadrement qui «devraient faciliter le transfert des ressources vers les secteurs où le Canada jouit d'un avantage comparatif à long terme, minimiser le coût de la libération des échanges en termes de chômage, de désorganisation industrielle et de déséquilibre régional, et enfin, assurer que les avantages que le Canada en retirera ne seront pas ob- tenus aux dépens de régions ou de groupes sociaux particuliers»(p. 180).

La base théorique de la principale conclusion du rapport est l'idée que sous des conditions très particulières reliées à la structure des marchés, au mouvement des facteurs et aux conditions de production, le libre-échange plus des transferts «optimaux» entre les gagnants et les perdants d'une telle politique assurent une production et une consommation mondiale maximale. Une telle conclusion ne s'applique pas nécessairement à un pays ou à une province en particulier, ni à un sous ensemble de personnes ou de facteurs à l'intérieur d'un pays. De plus, la conclusion précédente doit être sérieusement modifiée et pondérée lorsque la réallocation des revenus est difficile à effectuer ou lorsque nous introduisons des imperfections dans la concurrence, des revirements dans les intensités des facteurs de production ou encore des incertitudes dans le comportement de certains agents économiques. Cette base théorique est implicite dans le rapport même s'il n'effectue pas (ou si peu) les pondérations mentionnées. Une fois cette base théorique acceptée, le Conseil arrive à la conclusion qu'étant donné le nouvel ordre économique international, la croissance des blocs commerciaux (le Canada étant l'un des seuls pays industriels à ne pas avoir accèss à un marché de plus de cent millions d'habitants), l'apparition de nouveaux centres de croissance sur la base de la théorie du cycle des produits de Raymond Vernon, les sociétés multinationales, les relations économiques entre l'Est et l'Ouest, et les pénuries de produits essentiels, qu'une politique de libreéchange est la seule option commerciale possible pour le Canada. Pour s'accaparer des bénéfices de ce nouvel crdre économique, le Canada devra donc «transférer les ressources utilisées dans la production d'articles de technologie courante comportant un coefficient de travail élevé, vers des secteurs où ils jouissent d'un meilleur avantage relatif, 
en raison d'une technologie plus avancée et d'une main-d'oeuvre plus instruite ». (p. 64).

Une présentation sommaire du rapport étant donnée, nous tenterons dans les pages qui vont suivre d'effectuer une évaluation de trois points importants qui requièrent encore des éclaircissements de la part du Conseil.

a) Le rapport part d'une constatation pouvant difficilement être mise en doute: le secteur manufacturier canadien est handicapé par une faible productivité relative, faiblesse causée par l'absence d'échelle et de spécialisation. Le raisonnement du Conseil devient moins évident lorsqu'il affirme que les causes mentionnées sont uniquement ou à très forte majorité reliées à la politique protectionniste canadienne qui protégeait le secteur manufacturier contre la concurrence étrangère (d'où l'absence de spécialisation) et à la politique commerciale de nos clients qui fermaient la porte à nos exportations (d'où le manque d'échelle appropriée pour atteindre l'efficacité). Malgré les effets négatifs indéniables de ces barrières artificielles, leur importance ne doit pas être exagérée. Les désavantages comparés canadiens sont aussi dûs à des causes internes beaucoup moins artificielles telles la qualité et le nombre de ses entrepreneurs, l'immobilité relative de ses facteurs de production dûe à ses caractéristiques géo-politiques, le très faible niveau de dépenses dans les facteurs $\mathrm{R} \& \mathrm{D}$ et l'abondance trop sécurisante de ses matières premières, le manque d'innovation relié à la non complémentarité de sa structure économique régionale et industrielle, etc... Ces causes non artificielles, et combien d'autres encore, sont aussi responsables du manque de spécialisation, la politique protectionniste canadienne n'étant qu'un autre facteur décourageant l'innovation de techniques de production et le développement de produits manufacturés concurrentiels.

Quant à la politique commerciale des autres pays qui élimine ou réduit nos avantages comparés pour certains produits manufacturés sur leurs marchés locaux, son influence ne doit pas cacher l'effet d"autres va,riables comme les coûts de transport reliés à notre situation géographique, la gamme des produits exportés et la faible qualité des services après vente. Ces caractéristi- ques semblent moins affecter certains concurrents du Canada sur le marché américain et européen malgré le fait qu'ils doivent affronter les mêmes barrières commerciales pour les produits manufacturés et qu'ils ont une taille économique similaire à celle du Canada (pensons aux pays scandinaves, à la Suisse, à Taiwan, etc....)

Notre énumération de variables autres qu'artificielles désire simplement démontrer que les succès commerciaux d'une région sont aussi fonction de la qualité de ses hommes et de caractéristiques distinctes de sa politique commerciale. Même si une politique libreéchangiste «peut stimuler » la productivité manufacturière par l'élargissement de l'échelle de production et par la concurrence accrue, le Conseil Economique ne devrait pas s'attendre à ce qu'elle effectue des miracles. L'expérience japonaise et européenne nous a enseigné qu'une politique de libre-échange, ou même une libération graduelle des échanges dans le cadre des négociations du G.A.T.T., accélère le taux de croissance commerciale d'une région; elle n'est pas suffisante par contre pour permettre à elle seule un décollage du secteur manufacturier.

b) Notre deuxième point concerne les effets spécifiques ou particuliers d'une politique de libre-échange. En effet, le rapport ayant démontré que les tarifs, quotas et autres barrières commerciales distortionnent la structure mondiale (et canadienne) de production et de commerce, tout en affaiblissant le revenu réel, comment expliquer que les relations économiques internationales ne soient pas plus teintées de libéralisme commercial? La réponse est simple: la base théorique explicitée au début insistait sur le caractère général de la conclusion et laissait sous-entendre qu'un pays en particulier pouvait améliorer à court terme sa situation économique par une politique protectionniste (améliorant ses termes d'échanges, réduisant le chômage, encourageant l'industrialisation, ...) Il existe donc des coûts, un manque à gagner ou des désavantages spécifiques reliés au libreéchange non seulement au niveau des pays mais aussi au niveau de certains groupes particuliers dont les facteurs de production rares à l'intérieur d'une économie et les producteurs domestiques en concurrence avec les producteurs étrangers. Ces deux groupes, identifiés 
par le Conseil au chapitre 2, mettront tout en oeuvre pour bloquer la démarche vers le libre-échange parce qu'ils risquent de perdre le bien-être économique assuré artificiellement par le protectiunnisme. Tout au moins ils exigeront des compensations durant la période de transition: paiements d'assurance-chômage et autre assistance financière pour la main-d'oeuvre déplacée, subventions et allègements fiscaux pour les industries manufacturières devant disparaître, «restructurer leur production, développer de nouvelles lignes de produits et mettre sur pied de nouvelles facilités de commercialisation à l'étranger » (p. 186). Ces coûts ne seront pas négligeables, surtout en période de stagnation, et même si l'on peut escompter des bénéfices nets positifs d'une politique libre-échangiste, le rapport aurait dû leur accorder une attention plus importante que celle manifestée au chapitre 13. De plus il aurait intérêt à expliciter d'avantage la recommandation 18 qui paraît aux yeux des lecteurs des plus nébuleuses.

c) Qu'en est-il en terminant des effets régionaux d'une politique libreéchangiste canadienne? Le rapport du Conseil Economique est très explicite à ce sujet: «En régime de libre-échange unilatéral ou multilatéral, c'est au Québec d'abord, et en Ontario ensuite, que l'emploi diminuerait le plus, là encore parce que c'est dans cette dernière province que se concentrent les industries faisant concurrence aux importations, et qu'au Québec une forte proportion des industries ont un haut coefficient de main-d'oeuvre » (p. 186). Ces anticipations facilement prévisibles à cause des raisons citées ci-haut demandent donc des mesures spécifiques d'aide gouvernementale et des politiques d'encadrement pour faciliter le transfert des ressources. Or encore là, le rapport du Conseil pèche par optimisme. Il suggère l'implantation «de nouvelles industries ayant un avenir à long terme dans les régions menacées par la concurrence des importations » (p. 192); ceci demeure un voeu pieux surtout lorsqu'on regarde les résultats des programmes actuels, pourtant nombreux et bien pourvus. La même remarque s'applique à sa proposition d'une étroite collaboration fédérale-provinciale «pour assurer la cohésion de tous les aspects de l'aide canadienne à l'adaptation" (p. 193). Enfin, lorsqu'il avance l'idée que "ces diminutions de production et d'emploi se- raient toujours atténuées par toute dépréciation de la valeur du dollar canadien » (p. 185), il semble oublier un instant qu'une telle éventualité ferait augmenter le prix général des importations, résultat qui contrebalancerait un des effets majeurs d'une politique de libre-échange.

Nous avons répondu dans ce court texte au désir du Conseil Economique d'activer les discussions publiques autour d'une politique aussi importante pour l'avenir du Canada. Nos remarques sont demeurées pourtant partielles ne faisant pas ressortir les innombrables qualités et richesses d'informations contenues dans ce rapport. A ce titre, le document "Au-delà des frontières》 ainsi que les études spéciales qu'il résume, méritent amplement d'être lus.

\section{Carmine NAPPI}

Ecole des Hautes Etudes

Commerciales de Montréal

Le management d'aujourd'hui, Savoir organiser, Savoir décider, par Gérald Lefebvre, Montréal, Les Editions de l'Homme, 1975, $166 \mathrm{p}$.

Voici un volume à lire. A lire par les administrateurs, à lire par les étudiants concernés par le sujet et à lire par les théoriciens et professeurs pour y découvrir comment on peut rendre simples et opérationnelles diverses théories du management.

Dans la première partie, «Les notions de base », Lefebvre nous introduit à son conception de la gestion: "gérer, c'est gouverner ou veiller à ce que tout fonctionne pour le mieux, » (p. 20). "Pour gérer, il faut prévilégier certains éléments de la réalité au détriment des autres, il faut simplifier» (p. 22). On ne peut mieux illustrer le style simple et sans prétention de l'auteur. Derrière cette apparente simplicité se retrouve une profonde compréhension et intégration des sujets discutés qui en permettent une présentation claire et concrète.

Dans cette première partie, le chapitre trois, «L'organisation : un système ouvert », est une excellente synthèse et clarification de l'approche-système appliquée à l'organisation. Il est facile de sophistiquer cette approche de façon à éloigner plus d'un lecteur. Lefebvre fait exactement le contraire. Il manifeste un 